\title{
Effect of the Resveratrol Rice DJ526 on Longevity
}

\author{
Md. Saidul Islam ${ }^{1,+}$, Yan Yan Jin ${ }^{1,+}$, Hea-Jong Chung ${ }^{1,+}$, Hyeon-Jin Kim ${ }^{2}$, So-Hyeon Baek ${ }^{3, *}$ \\ and Seong-Tshool Hong $1, *$ \\ 1 Department of Biomedical Sciences and Institute for Medical Science, Chonbuk National University Medical \\ School, Jeonju, Chonbuk 54907, Korea \\ 2 BDRD Institute, JINIS Biopharmaceuticals Co., Bongdong, Wanju, Jeonbuk 55321, Korea \\ 3 Department of Well-Being Resources, Sunchon National University, Suncheon, Jeonnam 57922, Korea \\ * Correspondence: baeksh@scnu.ac.kr (S.-H.B.); seonghong@chonbuk.ac.kr (S.-T.H.); \\ Tel.: +82-61-750-3217 (S.-H.B.); +82-63-270-3105 (S.-T.H.) \\ + These three authors contribute equally to this paper.
}

Received: 3 July 2019; Accepted: 1 August 2019; Published: 5 August 2019

\begin{abstract}
Resveratrol is the best-known chemical for extending the lifespan of various organisms. Extensive recent research has shown that resveratrol can extend the lifespan of single-celled organisms, but its effects on the extension of animal lifespans are marginal. Despite the limited efficacy of pure resveratrol, resveratrol with the endogenous property of the DJ rice in the resveratrol rice DJ526 previously showed profound health benefits. Here, we report that the resveratrol rice DJ526 markedly extended the lifespan of the fruit fly Drosophila melanogaster by as much as $41.4 \%$ compared to that of the control. The resveratrol rice DJ526 also improved age-related symptoms such as locomotive deterioration, body weight gain, eye degeneration and neurodegeneration in $D$. melanogaster upon aging. This result shows the most significantly improved lifespan in animal experiments to date, meaning that the resveratrol rice DJ526 will assist in the development of a therapeutic agent for longevity or addressing age-related degeneration.
\end{abstract}

Keywords: resveratrol; lifespan; the resveratrol rice DJ526; Drosophila

\section{Introduction}

Human aging occurs through the gradual deterioration of the body over time. Naturally, this deterioration of the body makes aging the greatest risk factor for most human diseases [1,2]. Although addressing aging has been the primary scientific pursuit of modern health care, there is not much progress in understanding the aging process and anti-aging management. In this context, it is not surprising that many serious modern researchers have aimed to find a solution for anti-aging management.

Resveratrol (3, 5, 4' -trihydroxy-trans-stilbene) is a polyphonic bioflavonoid phytonutrient and an antioxidant produced by plant roots and fruits $[3,4]$. Although the identity of resveratrol has been well known for decades, the compound did not receive scientific attention until it was shown to extend the lifespan of Saccharomyces cerevisiae by stimulating the silent information regulator (Sir) [5-8]. Despite its excellent anti-aging effect in yeast, the therapeutic efficacy of resveratrol showed diminished reliability in animal studies [9-11]. Resveratrol also extended the lifespan of Caenorhabditis elegans through a Sir-2.1-dependent mechanism that inhibited the action of endoplasmic reticulum (ER) stress genes [12]. In contrast, resveratrol did not extend the lifespan of C. elegans under normal conditions but extended the lifespan under acute oxidative stress conditions in a dose-dependent manner [13]. Besides, resveratrol and other sirtuin-activating compounds (STACs) extended the lifespan of C. elegans and Drosophila melanogaster in a Sir2-dependent manner by mimicking caloric restriction [14], while another study reported that dietary resveratrol supplementation did not exert its beneficial lifespan 
extension effect on D. melanogaster [15,16]. In an animal model, resveratrol altered the physiology of mice on a high-calorie diet, showing improved health and a slight extension of lifespan $[11,17,18]$. By contrast, long-term resveratrol administration failed to extend the lifespan of mice while it slowed down age-related degeneration with similar changes to the gene expression patterns induced by dietary restriction $[19,20]$. The aforementioned findings have been suggested to indicate that resveratrol extends the lifespan of single-celled organisms such as Saccharomyces cerevisiae, but its effects on lifespan extension in animals were very limited.

Previously, we developed the resveratrol rice DJ526 by transferring the resveratrol biosynthesis gene, stilbene synthase, from the Arachis hypogaea (peanut) variety Palkwang into Oryza sativa (rice) japonica variety Dongjin (DJ), which accumulated a significant amount of resveratrol, at 1.4-1.9 $\mu \mathrm{g} / \mathrm{g}$, in its grains [21-24]. The resveratrol rice DJ526 showed significant health-benefits on obesity and related metabolic syndrome through the synergistic effect of resveratrol with the endogenous property of the DJ rice compared to the resveratrol or control, which had minimal effects [21-24]. The therapeutic efficacies of the resveratrol rice DJ526 on obesity and related metabolic syndrome were comparable to those of typical pharmaceutical drugs [21-24].

Considering that the original efficacy of resveratrol is anti-aging, it would be worthwhile to investigate the anti-aging efficacy of resveratrol rice DJ526 as human food. In our recent study, we investigated the longevity effect of DJ526 callus induced from the mature seeds of the resveratrol DJ526 rice, demonstrating that the callus of the resveratrol rice DJ526 could extend the lifespan of Drosophila melanogaster [25]. In continuation of our longevity study with DJ526, it is worthwhile to investigate the edible part of rice, the grains from DJ526 rice. In this study, we investigate the anti-aging effects of the resveratrol rice DJ526 using grains to feed D. melanogaster. Our experimental results show that the resveratrol rice DJ526 markedly extends D. melanogaster lifespan by as much as $41.4 \%$ compared to the control. This degree of lifespan extension of an experimental animal is the most significant extent to date compared to other therapeutic agents. We believe that the resveratrol rice DJ526 deserves further attention and study for its possible therapeutic use for longevity or age-related diseases, as well as its contribution to elucidating the aging process.

\section{Materials and Methods}

\subsection{Drosophila Strains and Maintenance}

The wild-type Harwich strain (FBst0004264) of D. melanogaster was obtained from the Bloomington Drosophila Stock Center (Indiana University, Bloomington, IN, USA) and the wild-type ORR strain of D. melanogaster was provided by Isaac A. Adedara (Federal University of Santa Maria, Santa Maria, RS, Brazil). The D. melanogaster individuals used in these experiments were routinely maintained at $18{ }^{\circ} \mathrm{C}$ on standard cornmeal media in a $60 \%$ humidified incubator with a $12 \mathrm{~h}$ light-12 h dark cycle.

\subsection{Drosophila Experiments}

The D. melanogaster maintained on the standard cornmeal media at $25^{\circ} \mathrm{C}$ were divided into five groups to transfer onto standard cornmeal media (Ctrl), cornmeal media supplemented with resveratrol (RS) media, cornmeal media supplemented with DJ rice (DJ), DJ media supplemented with resveratrol (DJRS), and cornmeal media supplemented with resveratrol rice DJ526 (DJ526) for laying eggs (Table S1), and the fly larvae were maintained in the media at $25^{\circ} \mathrm{C}$. Within a few hours of eclosion, the flies were collected under brief $\mathrm{CO}_{2}$ anesthesia and incubated at $25^{\circ} \mathrm{C}$ for $48 \mathrm{~h}$ for maturation. The mature flies were transferred to the respective diets as indicated above and cultured at $18^{\circ} \mathrm{C}$ in a humidified incubator under a $12 \mathrm{~h} \mathrm{light-12} \mathrm{h}$ dark cycle. After the fly maturation, the rest of the fly experiments were performed at $18{ }^{\circ} \mathrm{C}$. Each of the 150 adult male and 150 adult female flies were used for the lifespan assay. A vial large enough for 50 flies was used to avoid over-crowding. The flies were transferred to fresh vials with fresh media every 4 days until all flies were dead. The number of dead flies was recorded, and the survival data were analyzed using the Kaplan-Meier method. 
The Kaplan-Meier estimate is the non-parametric maximum likelihood of survival at a given time point $S(t)$ as follows:

$$
\mathrm{S}(\mathrm{t})=\prod_{\mathrm{ti}<\mathrm{t}} \frac{\mathrm{ni}-\mathrm{di}}{\mathrm{ni}} .
$$

where $n i$ is the number of survivors minus the number of censored cases and $d i$ is the number of deaths at time point $t i$ [26].

\subsection{Locomotion Assay}

The adult male and female flies post-eclosion with the same starting age were on the experimental diets as indicated. For the locomotion assays, 15 flies from each group were maintained in a plastic Drosophila culture vial and the media were changed every 4 days. The flies in the plastic tube were anaesthetized with $\mathrm{CO}_{2}$ by being placed into $15-\mathrm{mL}$ empty plastic tubes, which were bunged with cotton wool to prevent escape, at room temperature. The flies were recovered from $\mathrm{CO}_{2}$ exposure for $30 \mathrm{~min}$ before the assay. After gentle tapping, the flies at the bottom of the tube were allowed to climb a height of $10 \mathrm{~mL}$ within $30 \mathrm{~s}$ for the observation of their climbing performances. The number of flies at the top, above the 10-mL mark of the tube, and at the bottom, below the 2-mL mark of the tube, was recorded after $30 \mathrm{~s}$. Three trials were performed for each respective diet at each time point. The climbing abilities of the flies were observed at the 10th, 30th, 60th and 90th days post-eclosion. The total number of flies used in each group was 100. The performance index (PI) was calculated for each wild-type Drosophila group of 15 flies using the formula PI $=0.5 \times\left(n_{\text {total }}+n_{\text {top }}-n_{\text {bottom }}\right) / n_{\text {total }}$, where $n_{\text {total }}$ is the total number of flies, $n_{\text {top }}$ is the total number of flies at the top, and $n_{\text {bottom }}$ is the total number of flies at the bottom [27].

\subsection{Body Weight Measurements}

Newly eclosed fresh adult male and female flies were fed the experimental media as indicated. The body weights of the individual adult flies were measured at the 10th, 30th, 60th and 90th days post-eclosion.

\subsection{Light Microscopy of the Drosophila Eye}

Adult male and female flies were fed respective diets and maintained as described above. The flies were collected on the indicated days post-eclosion. Ten male and 10 female flies of the WT ORR and WT Harwich strains from each respective media were anaesthetized with $\mathrm{CO}_{2}$ and transferred into the Eppendorf tube. The flies were then frozen at $-80^{\circ} \mathrm{C}$ for $1 \mathrm{~h}$ and mounted on their sides, and their eyes were examined using a dissecting light microscope (Amscope, ZM-4TW3-FOR-8M, Irvine, CA, USA) equipped with an Olympus SZ51 lamp; the eye images were captured with a microscopic camera (Amscope, MU-1000). The eye images of flies were analyzed for each respective diet at the indicated days post-eclosion.

\subsection{Histological Examination of the Drosophila Brains}

The adult male and female flies were fed respective types of diets and maintained as described above. The flies were collected on the 10th, 30th, 60th and 90th days post-eclosion for histological examination. After anesthesia with $\mathrm{CO}_{2}$, the flies were kept at $-80^{\circ} \mathrm{C}$ for $1 \mathrm{~h}$. The fly heads were collected and fixed in 10\% neutral buffered formalin (Sigma Aldrich, St. Louis, MO, USA) at room temperature, followed by embedding in paraffin using standard histological procedures. The embedded heads were sectioned at $6 \mu \mathrm{m}$ for histological examination. The paraffin in the brain section on glass microscopic slides was removed through washing with hot water. Then, the slides were air-dried and baked overnight at $65^{\circ} \mathrm{C}$. The brain sections were stained with haematoxylin and eosin. The stained brain images were taken under a slide scanner microscope by Apero Scan Scope FL (Leica Biosystems, Nussloch, Germany) at a magnification of 10x; scale bars, $200 \mu \mathrm{m}$. 


\subsection{Statistical Analysis}

An analysis of the survival data was performed using the Kaplan-Meier method with data preparation using Graph Pad Prism version 8.1.2 software (GraphPad Software, Inc. San Diego, CA, USA). All comparisons were made with log-rank tests (Figure 1). The statistical analysis was expressed as the means \pm standard deviation (s.d.) as indicated. The significant differences between the two groups were analyzed with an unpaired Student's $t$-test, being considered statistically significant if $p<0.05$. The statistical significance was shown as ${ }^{*} p<0.05,{ }^{* *} p<0.01$ and ${ }^{* * *} p<0.001$ from three independent experiments.
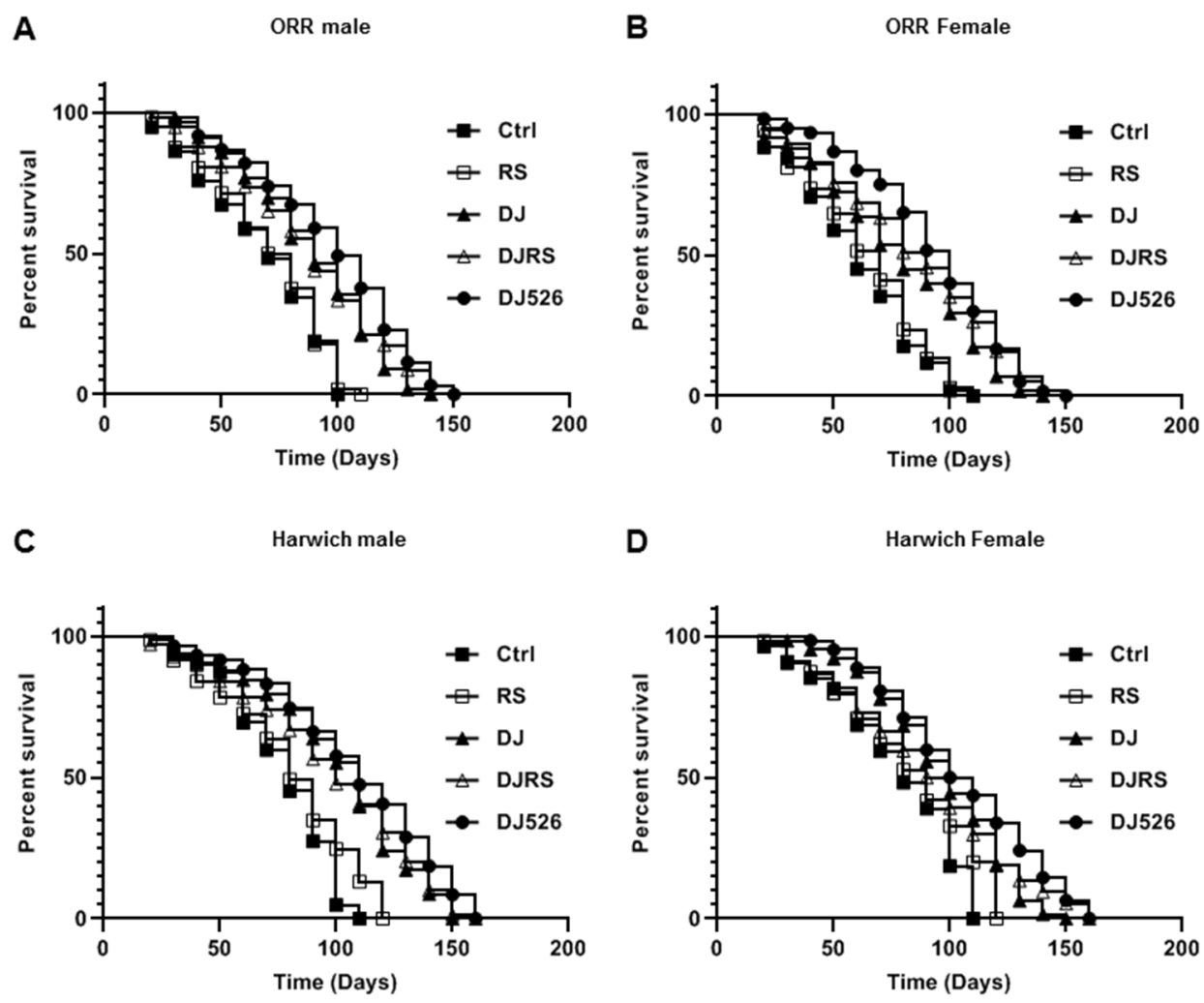

Figure 1. The resveratrol rice DJ526 increased the lifespan of Drosophila melanogaster. (A) ORR males, (B) ORR females, (C) Harwich males and (D) Harwich females. Ctrl represents standard cornmeal medium; RS represents cornmeal medium supplemented with resveratrol at $31.54 \mu \mathrm{g} / \mathrm{L}$, the equivalent amount of resveratrol found in the DJ526 media; DJ represents the medium in which $50 \%$ of cornmeal was replaced with Dongiin rice; DJRS represents the medium in which $50 \%$ of cornmeal was replaced with Dongiin rice and supplemented with resveratrol at $31.54 \mu \mathrm{g} / \mathrm{L}$; and DJ526 represents the medium in which $50 \%$ of the cornmeal was replaced with the resveratrol rice DJ526 (Table S1). For the lifespan assay, the survival rate of 150 flies from each group was monitored with medium change every 4 days. Comparisons were made using log-rank tests. The $p$ values (log-rank tests) for each strain and each sex were as follows. (A) ORR male flies: DJ526 versus Ctrl $(p<0.0001)$, RS $(p<0.0001)$, DJ $(p=0.0228)$, and DJRS $(p=0.1074)$, respectively. (B) ORR female flies: DJ526 versus Ctrl $(p<0.0001)$, RS $(p<0.0001)$, DJ $(p=0.0259)$, and DJRS ( $p=0.4557)$, respectively. (C) Harwich male flies: DJ526 versus Ctrl $(p<0.0001)$, RS $(p<0.0001)$, DJ $(p=0.0721)$, and DJRS ( $p=0.0788)$, respectively. (D) Harwich female flies: DJ526 versus Ctrl $(p=0.0001)$, RS $(p<0.0001)$, DJ $(p=0.0219)$, and DJRS $(p=0.0794)$, respectively. The percentage of surviving flies is shown along with the maximum lifespan in each group $(n=150)$. 


\section{Results}

\subsection{The Resveratrol Rice DJ526 Dramatically Extended the Median Lifespan of D. melanogaster}

To evaluate the efficacy of the resveratrol rice DJ526 on lifespan extension in fly, two different wild-type (WT) strains of D. melanogaster, ORR and Harwich, were used in this study. Feeding experiments were conducted by feeding WT Drosophila five different diets; a control diet with standard cornmeal diet of Drosophila; a DJ diet, in which $50 \%$ of the cornmeal in the standard cornmeal diet was replaced with the DJ rice; a DJ526 diet, in which $50 \%$ of the cornmeal was replaced with the resveratrol rice DJ526; an RS diet, in which $31.54 \mu \mathrm{g} / \mathrm{L}$ resveratrol, the equivalent amount found in the resveratrol rice DJ526, was supplemented to the standard cornmeal diet; a DJRS diet, in which $50 \%$ of the cornmeal was replaced with the DJ rice with supplementation of resveratrol at $31.54 \mu \mathrm{g} / \mathrm{L}$ (Table S1).

The effect of the resveratrol rice DJ526 on lifespan was evaluated by recording the dead flies with aging (Figure 1). In concordance with previous publications on the effect of resveratrol, the median lifespan increment of the RS groups (D. melanogaster was given the diet containing an equivalent amount of resveratrol as that of the DJ526 group) was not significant compared to the control groups (D. melanogaster receiving a standard cornmeal diet). The survival rate differences between each group became evident from the 30th day of the feeding experiments. The median lifespans of the ORR flies in the DJ526 groups were 152 days for males and 156 days for females, which were most extended compared to the control groups (108 days for males and 112 days for females), RS groups (116 days for both males and females), DJ groups (140 days for males and 144 days for females), and DJRS groups (144 days for males and 148 days for females). In log-rank tests, the resveratrol rice DJ526 significantly extended the median lifespan of ORR flies compared to the control groups $(p<0.0001$ for both male and female), RS groups ( $p<0.0001$ for both male and female) DJ groups ( $p=0.0228$ for male and $p=0.0259$ for female), and DJRS groups ( $p=0.1074$ for male and $p=0.4557$ for female) (Figure 1A,B).

Similar to the ORR flies, the median lifespans of the Harwich flies in the DJ526 groups were 164 days for both males and females, which was extended most compared to the control groups (116 days for both males and females), RS groups (120 days for males and 128 days for females), DJ groups (156 days for both males and females), and DJRS groups (156 days for males and 160 days for females). In addition, log-rank tests showed that the resveratrol rice DJ526 significantly extended the median lifespan of Harwich flies also, compared to the control groups $(p<0.0001$ for both male and female), RS groups ( $p<0.0001)$, DJ groups ( $p=0.0721$ for male and $p=0.0219$ for female only), and DJRS groups ( $p=0.0788$ for male and $p=0.0794$ for female) (Figure 1C,D). Overall, the resveratrol rice DJ526 increased the median lifespan by as much as $40.7 \%$ for ORR D. melanogaster and $41.4 \%$ for Harwich D. melanogaster, compared to the control (Figure 1). Overall, the maximum lifespan of the DJ526 group flies was increased significantly (Figure 1). These results clearly showed that the interaction of resveratrol and the endogenous characteristics of DJ rice in the resveratrol rice DJ526 synergistically extended the lifespan of $D$. melanogaster.

\subsection{The Resveratrol Rice DJ526 Ameliorated the Locomotive Deterioration of D. melanogaster during Age Progression}

Since a paucity of locomotion is a typical symptom of aging, we tested the flies' locomotive ability by measuring $D$. melanogaster climbing. The climbing ability of $D$. melanogaster was measured over their age progression to assess the effect of the resveratrol rice DJ526 (Figure 2). Its effect was noted starting from the 30th day in both D. melanogaster strains. The DJ526 groups of ORR strain climbed the test tube 1.52 times better than the control group, 1.42 times better than the RS group, 1.16 times better than the DJ group and 1.08 times better than the DJRS group at the 90th day after feeding. Also, the DJ526 groups of Harwich strain climbed the test tube 1.64 times better than the control group, 1.52 times better than the RS group, 1.21 times better than the DJ group and 1.11 times better than the DJRS group at the 90th day after feeding. These results indicated that the resveratrol rice DJ526 helped 
to ameliorate locomotive deterioration in D. melanogaster upon aging to maintain their locomotive ability, supporting the lifespan increment data in Figure 1.

A

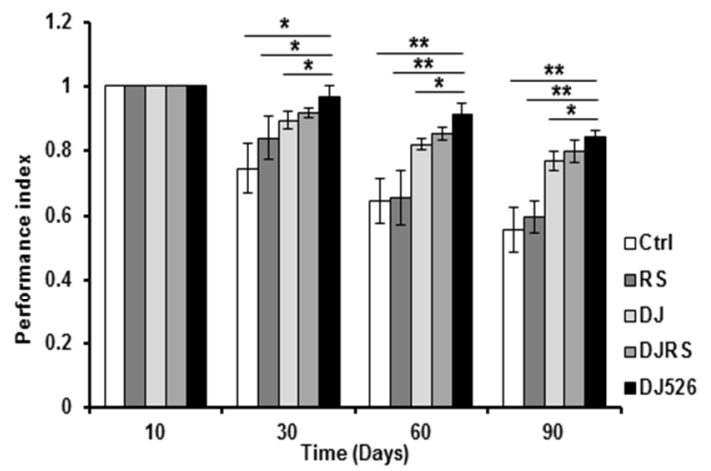

C

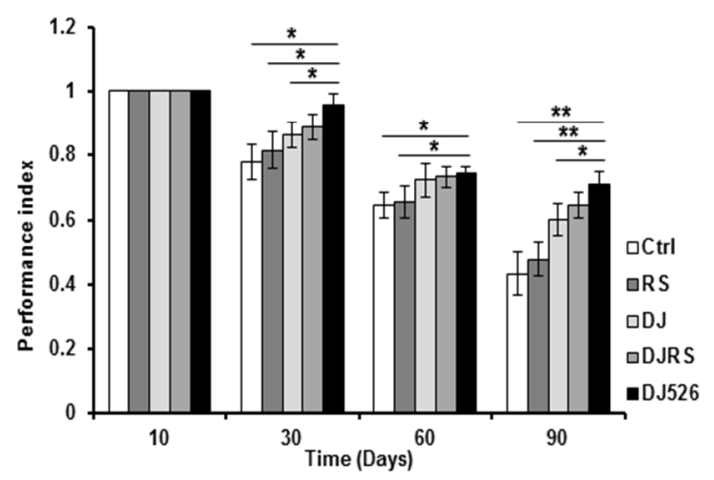

B

ORR Female

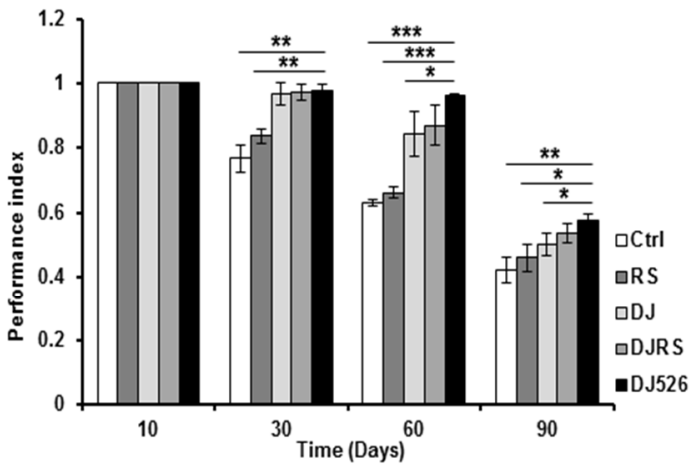

D

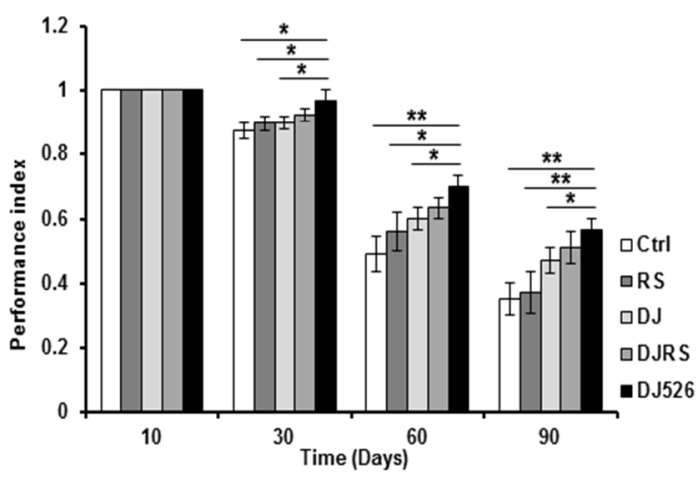

Figure 2. The resveratrol rice DJ526 improved the locomotion activity of D. melanogaster. (A) ORR males, (B) ORR females, (C) Harwich males and (D) Harwich females with age progression. Ctrl represents standard cornmeal medium; RS represents cornmeal medium supplemented with resveratrol at $31.54 \mu \mathrm{g} / \mathrm{L}$, the equivalent amount of resveratrol found in the DJ526 media; DJ represents the medium in which $50 \%$ of cornmeal was replaced with Dongjin rice; DJRS represents the medium in which $50 \%$ of cornmeal was replaced with Dongjin rice and supplemented with resveratrol at $31.54 \mu \mathrm{g} / \mathrm{L}$, and DJ526 represents the medium in which $50 \%$ of the cornmeal was replaced with the resveratrol rice DJ526 (Table S1). Fly locomotor activity was observed as indicated until 90th days post-eclosion and was indicated as the performance index. Statistical significance was analyzed with an unpaired Student's $t$-test and indicated as ${ }^{*} p<0.05,{ }^{* *} p<0.01$, and ${ }^{* *} p<0.001$ from three independent experiments $(n=15)$.

\subsection{The Resveratrol Rice DJ526 Caused D. melanogaster to Maintain a Healthy Body Weight during Age Progression}

Considering that weight gain is an important indicator of animal aging, we investigated the effect of the resveratrol rice DJ526 on the body weight of D. melanogaster. The body weights of all the fly groups gradually increased with age progression (Figure 3). However, as expected from its anti-aging property, the flies in the DJ526 groups did not show excessive weight gain during age progression (Figure 3). The body weights of D. melanogaster ORR in the DJ526 groups were $1.64 \pm 0.03 \mathrm{mg}$ for male flies and $1.74 \pm 0.01 \mathrm{mg}$ for female flies at the 90th day after feeding, which were significantly lower than those of the control groups (1.91 $\pm 0.07 \mathrm{mg}$ for males and $2.14 \pm 0.09 \mathrm{mg}$ for females), RS groups $(1.74 \pm 0.04 \mathrm{mg}$ for males and $2.11 \pm 0.09 \mathrm{mg}$ for females), DJ groups $(1.70 \pm 0.02 \mathrm{mg}$ for males and $1.82 \pm 0.03 \mathrm{mg}$ for females), and DJRS groups ( $1.70 \pm 0.01 \mathrm{mg}$ for males and $1.79 \pm 0.02 \mathrm{mg}$ for females). 
A

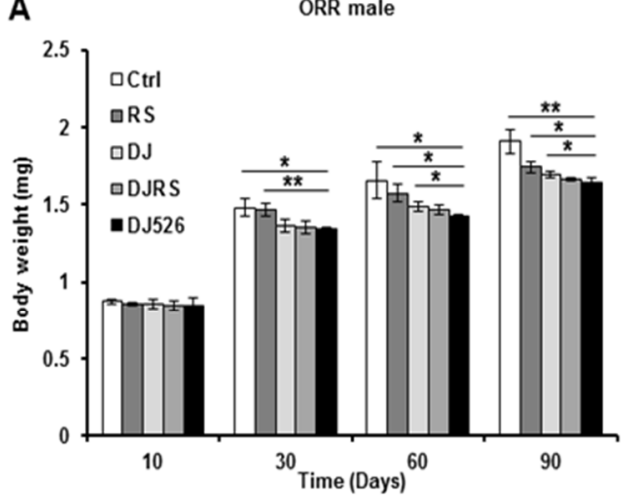

C

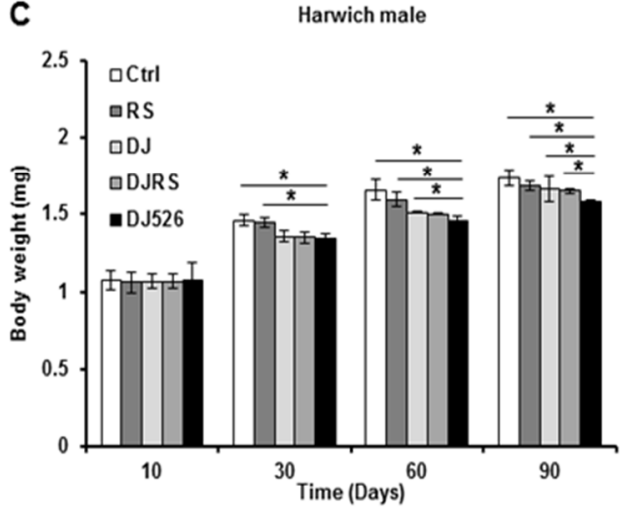

B

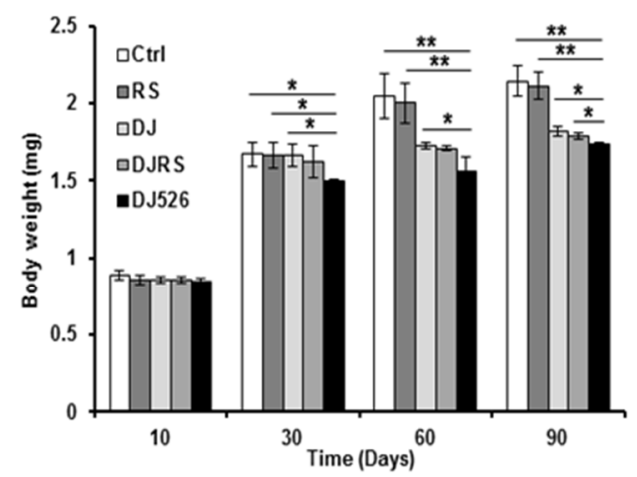

D

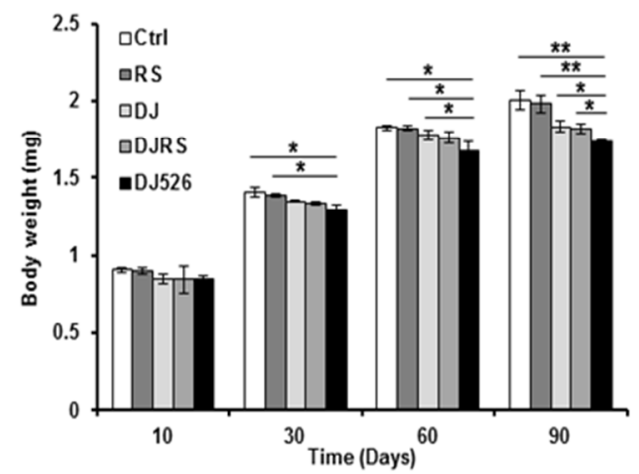

Figure 3. The effects of the resveratrol rice DJ526 on changes in the body weights of D. melanogaster. (A) ORR males, (B) ORR females, (C) Harwich males and (D) Harwich females with age progression. Ctrl represents standard cornmeal medium; RS represents cornmeal medium supplemented with resveratrol at $31.54 \mu \mathrm{g} / \mathrm{L}$, the equivalent amount of resveratrol found in the DJ526 media; DJ represents the medium in which 50\% of cornmeal was replaced with Dongin rice; DJRS represents the medium in which $50 \%$ of cornmeal was replaced with Dongjin rice and supplemented with resveratrol at $31.54 \mu \mathrm{g} / \mathrm{L}$, and DJ526 represents the medium in which $50 \%$ of the cornmeal was replaced with the resveratrol rice DJ526 (Table S1). The body weights were measured at the 10th, 30th, 60th and 90th days post-eclosion. Statistical significance was analyzed with an unpaired Student's $t$-test and indicated as ${ }^{*} p<0.05$ and ${ }^{* *}$ $p<0.01$ from 3 independent experiments $(n=30)$.

Similarly, the body weights of D. melanogaster Harwich in the DJ526 groups were $1.58 \pm 0.01 \mathrm{mg}$ for male flies and $1.74 \pm 0.01 \mathrm{mg}$ for female flies at the 90th day after feeding, which were significantly lower than the body weights of the control groups $(1.74 \pm 0.05 \mathrm{mg}$ for males and $2.00 \pm 0.06 \mathrm{mg}$ for females), RS groups (1.69 $\pm 0.03 \mathrm{mg}$ for males and $1.98 \pm 0.06 \mathrm{mg}$ for females), DJ groups $(1.67 \pm 0.08 \mathrm{mg}$ for males and $1.83 \pm 0.03 \mathrm{mg}$ for females), and DJRS groups ( $1.65 \pm 0.02 \mathrm{mg}$ for males and $1.82 \pm 0.03 \mathrm{mg}$ for females). Overall, the resveratrol rice DJ526 provided prominent health benefits relating to the maintenance of a healthy body weight compared to other groups of flies, supporting our recent study with DJ526 callus [25].

\subsection{The Resveratrol Rice DJ526 Inhibited Eye Degeneration in D. melanogaster during Age Progression}

Drosophila eye is known as an ideal model for investigating morphogenic changes, cell fate specification, and patterning [28]. We noticed that the morphological characteristics of the Drosophila eye accurately represented the degree of aging. The morphological characteristics of eye damage, roughness and the loss of eye pigment, were used to assess the severity of aging (Figure 4). The morphological observation of the Drosophila eyes clearly showed that the resveratrol rice DJ526 prevented eye degeneration during age progression. It should be noted that the eyes of WT flies, both 
the ORR and Harwich flies, under the DJ526 diet were rarely damaged, even at the 90th day after feeding, which was significantly different from the other groups of flies.

A
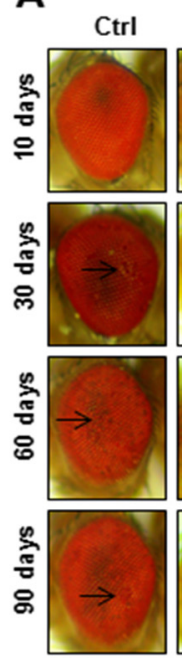

C
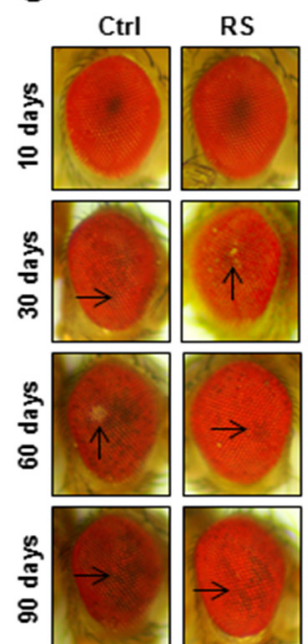

ORR Male

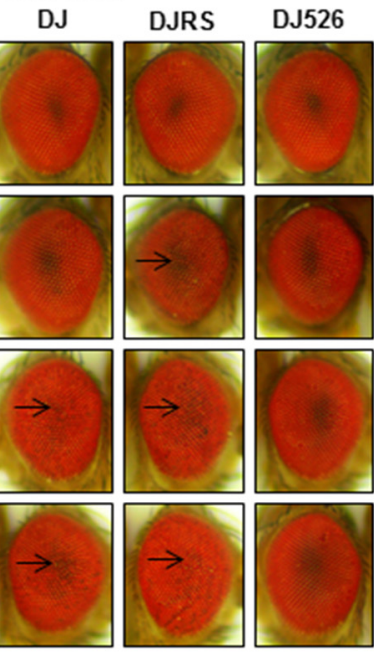

Harwich Male

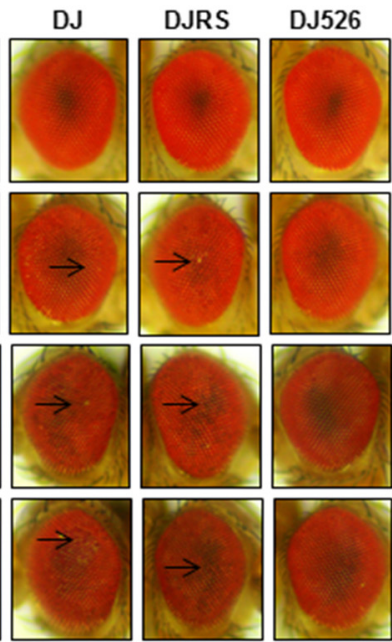

B
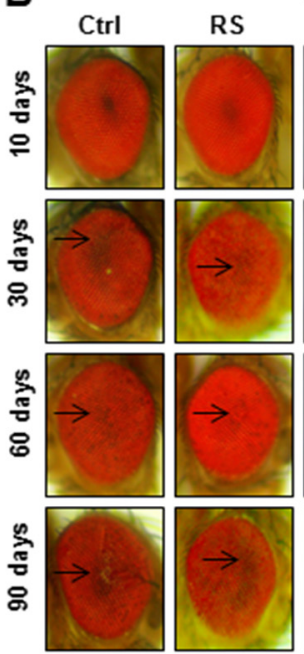

ORR Female

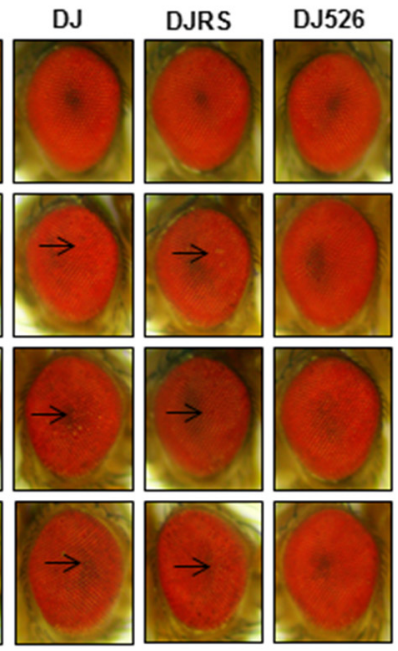

D

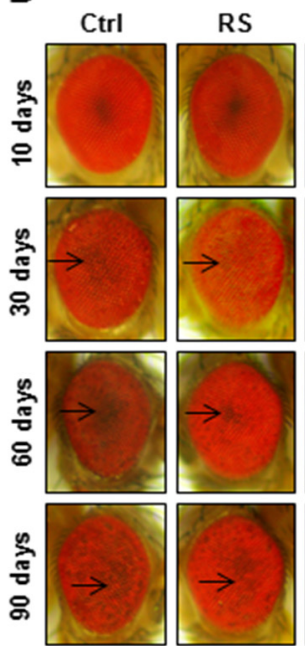

Harwich Female

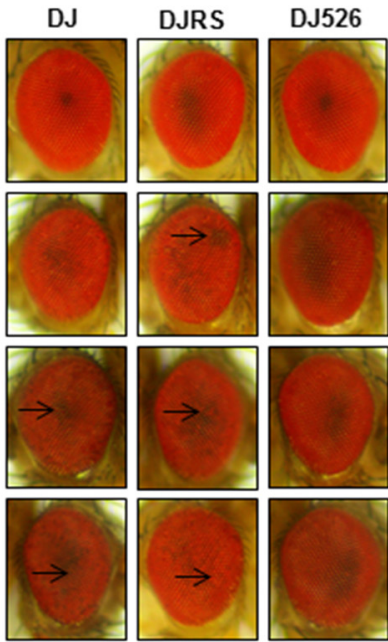

Figure 4. The resveratrol rice DJ526 suppressed the developmental eye defects in D. melanogaster. (A) ORR males, (B) ORR females, (C) Harwich males and (D) Harwich females with age progression. Ctrl represents standard cornmeal medium; RS represents cornmeal medium supplemented with resveratrol at $31.54 \mu \mathrm{g} / \mathrm{L}$, the equivalent amount of resveratrol found in the DJ526 media; DJ represents the medium in which $50 \%$ of cornmeal was replaced with Dongjin rice; DJRS represents the medium in which $50 \%$ of cornmeal was replaced with Dongjin rice and supplemented with resveratrol at $31.54 \mu \mathrm{g} / \mathrm{L}$, and DJ526 represents the medium in which $50 \%$ of the cornmeal was replaced with the resveratrol rice DJ526 (Table S1). Light microscopy studies of the Drosophila compound eyes were performed at the 10th, 30th, 60th and 90th days post-eclosion, and the eye damages are indicated as arrows.

\subsection{The Resveratrol Rice DJ526 Ameliorated Neurodegeneration in D. melanogaster during Age Progression}

Since DJ526 inhibited aging and ameliorated the aging symptoms in D. melanogaster (Figures 1-4), we also investigated whether the resveratrol rice DJ526 inhibits age-related neurodegeneration with age progression as well. The brains of each group of flies were isolated over the time course of the experiments, and we examined fly brain slices after haematoxylin and eosin (H\&E) staining. In the control groups, the vacuolar lesions became widespread as the fly age progressed, which can be used as a critical pathological marker of neurodegeneration in D. melanogaster (Figure S1, Figures 5 and 6). 
While the vacuolar lesions also appeared gradually and spread with age progression in the RS, DJ, and DJRS groups, the brains of the DJ526 group had significantly fewer vacuolar lesions compared to the other groups. The histological differences between each group became dramatically different at the 90th day after feeding. The brains of both fly strains in the DJ526 groups had few vacuolar lesions and maintained generally healthy brain integrity compared to the other groups. Additionally, it is notable that the brains of the RS, DJ, and DJRS groups showed fewer vacuolar lesions and maintained better brain integrity than the control group. The histological observation could be concluded to indicate that the resveratrol rice DJ526 offered excellent protection against neurodegeneration by suppressing the aging process.

A

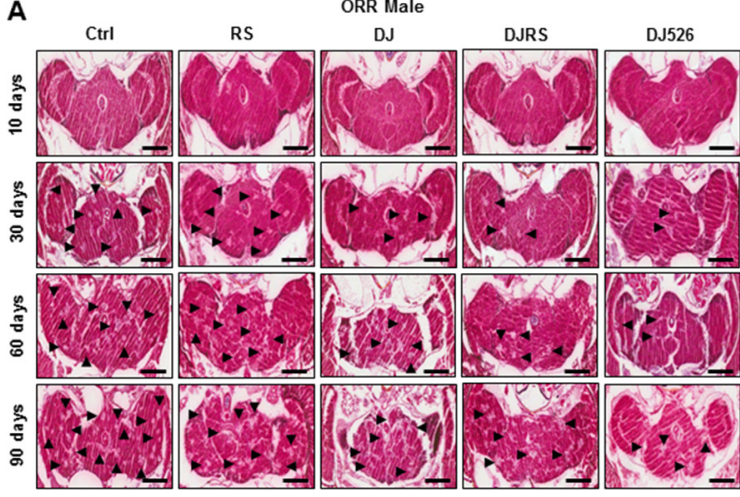

C

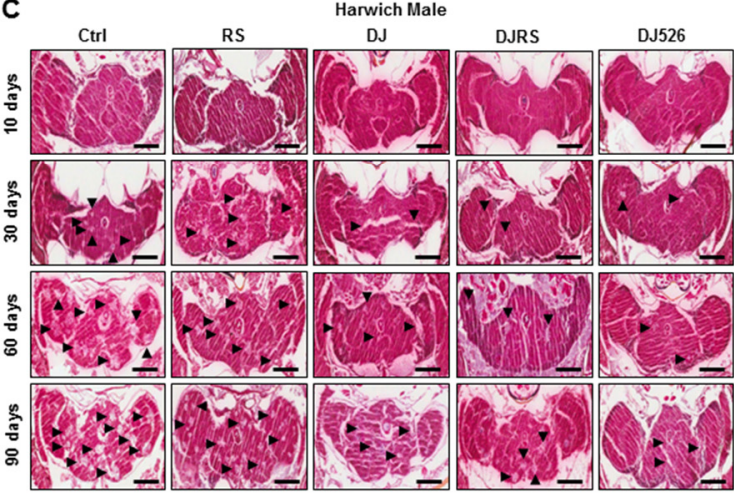

B
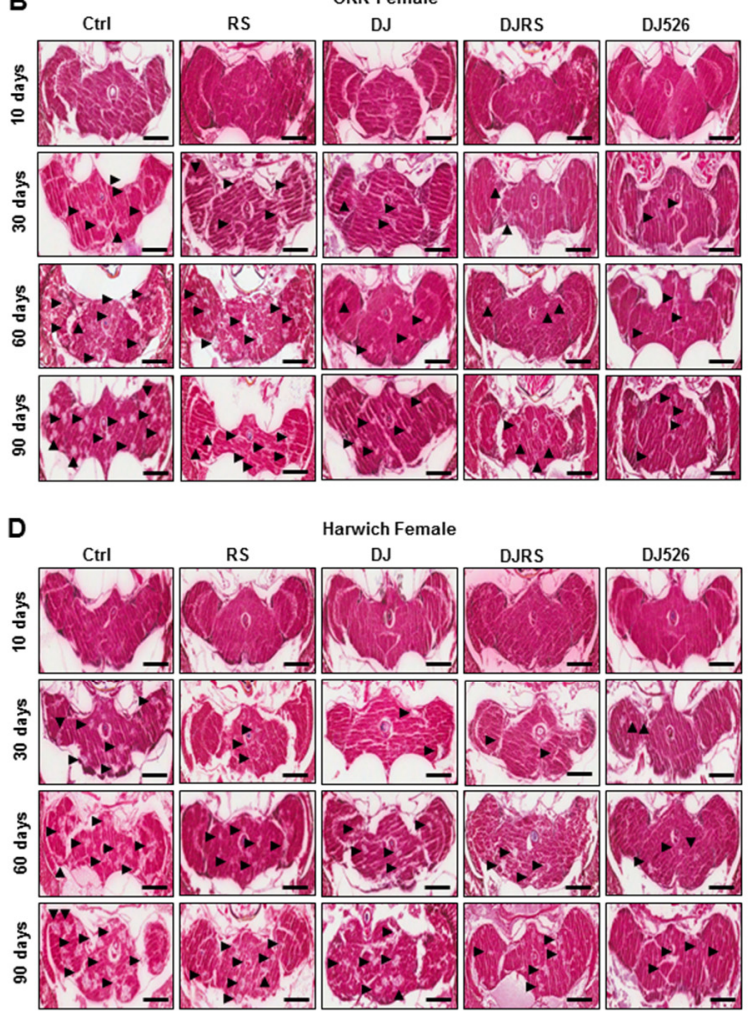

Figure 5. The resveratrol rice DJ526 inhibited age-related neurodegeneration in D. melanogaster. (A) ORR males, (B) ORR females, (C) Harwich males and (D) Harwich females. Ctrl represents standard cornmeal medium; RS represents cornmeal medium supplemented with resveratrol at $31.54 \mu \mathrm{g} / \mathrm{L}$, the equivalent amount of resveratrol found in the DJ526 media; DJ represents the medium in which $50 \%$ of cornmeal was replaced with Dongjin rice; DJRS represents the medium in which $50 \%$ of cornmeal was replaced with Dongjin rice and supplemented with resveratrol at $31.54 \mu \mathrm{g} / \mathrm{L}$, and DJ526 represents the medium in which $50 \%$ of the cornmeal was replaced with the resveratrol rice DJ526 (Table S1). A histological analysis was performed by H\&E staining to examine the neurodegeneration of the Drosophila brains at the 10th, 30th, 60th and 90th days post-eclosion. $n=100$; scale bars: $200 \mu \mathrm{m}$. The vacuolar lesions are indicated as black triangles. 
A

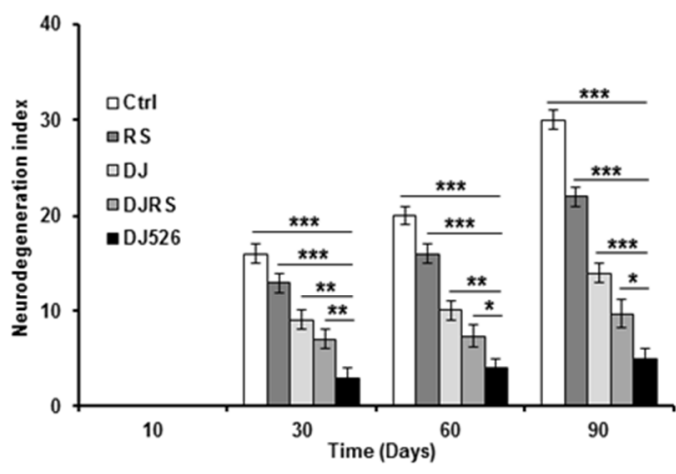

C

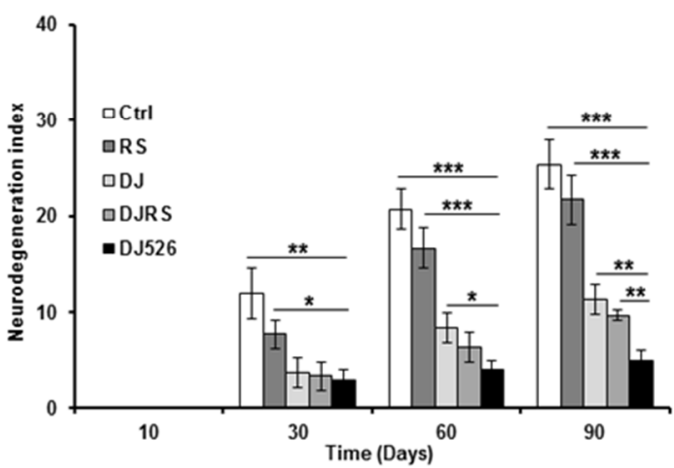

B

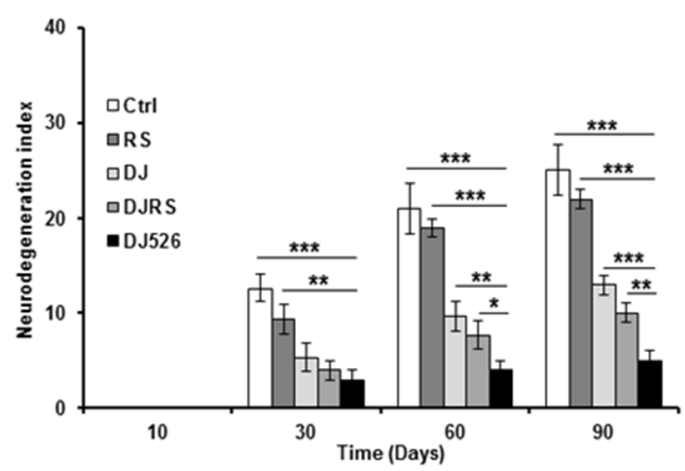

D

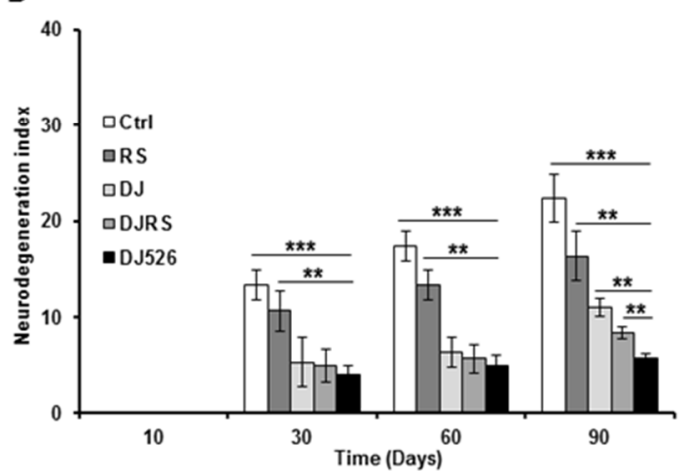

Figure 6. The resveratrol rice DJ526 suppressed age-related neurodegeneration in D. melanogaster. (A) ORR males, (B) ORR females, (C) Harwich males and (D) Harwich females. Ctrl represents standard cornmeal medium; RS represents cornmeal medium supplemented with resveratrol at $31.54 \mu \mathrm{g} / \mathrm{L}$, the equivalent amount of resveratrol found in the DJ526 media; DJ represents the medium in which $50 \%$ of cornmeal was replaced with Dongjin rice; DJRS represents the medium in which $50 \%$ of cornmeal was replaced with Dongjin rice and supplemented with resveratrol at $31.54 \mu \mathrm{g} / \mathrm{L}$, and DJ526 represents the medium in which $50 \%$ of the cornmeal was replaced with the resveratrol rice DJ526 (Table S1). The quantification of the neurodegeneration and vacuolar lesions based on the histological analysis of the Drosophila brains were observed at the 10th, 30th, 60th and 90th days post-eclosion. Statistical significance was analysed with an unpaired Student's $t$-test and indicated as ${ }^{*} p<0.05,{ }^{* *} p<0.01$, and *** $p<0.001$ from three independent experiments $(n=30)$.

\section{Discussion}

The biological process of aging has been well defined. The aging process, however, has been poorly understood with no effective prevention, treatment or therapy for aging [2,29]. Among the various attempts to increase the lifespan, caloric restriction (CR) has been the most effective in expansion of lifespan in diverse species from yeast to mammals [30-33]. Interestingly, resveratrol administration has been shown to mimic transcriptional aspects of CR in mice $[19,34]$. That characteristic of resveratrol gives us new hope for developing a longevity drug. However, resveratrol did not increase the lifespan despite delaying aging-related physiological deteriorations by modulating the gene expression of energy metabolism by mimicking CR $[3,19,35]$, which means that resveratrol itself is not sufficient as a therapeutic drug candidate for improving longevity or suppressing aging-related degenerative diseases.

Although the benefits of supplementary resveratrol are limited in animal experiments, our previous studies indicated that the transgenic resveratrol rice DJ526 provided unexpectedly high beneficial health effects through a synergistic mechanism for treating obesity and related metabolic syndrome $[21-24,36]$. However, those therapeutic efficacies have not been achieved through pure supplementary resveratrol treatment. This study showed that feeding $D$. melanogaster with the resveratrol rice DJ526 extended the fly lifespan by as much as $41.4 \%$. Although various studies are 
reporting the extension of the D. melanogaster lifespan [37-43], no chemicals or nutrients have been successfully extended the lifespan of D. melanogaster by as much as the resveratrol rice in this work.

The aging process is accompanied by the degeneration of almost all the tissues and organs, as well as the increase in the body weight $[1,44,45]$. This study showed that the resveratrol rice DJ526 not only increased the lifespan but also ameliorated the degeneration of the eye and brain of $D$. melanogaster. Also, the resveratrol rice DJ526 caused the flies to maintain a healthy body weight and locomotion. Considering that decreased locomotion and increased body weight represent important indicators of aging, it would be reasonable to conclude that the resveratrol rice DJ526 has a clear anti-aging effect. Overall, the resveratrol rice DJ526 could be an ideal functional food for treating and/or preventing aging, as well as various age-related diseases such as obesity, metabolic syndrome, etc.

In contrast to resveratrol rice DJ526 diet, the supplementation of resveratrol had almost no effect on its lifespan extension in D. melanogaster (Figure 1) despite its slight improvement in other age-related deterioration (Figures 2-6). These experimental results are in good agreement with previous studies showing that resveratrol supplementation has almost no effect or insignificant improvements on the lifespan of $D$. melanogaster $[15,16,46]$. In this context, this work and our previous research on resveratrol rice DJ526 shines a light on the new therapeutic candidate for anti-aging and longevity. In addition, our experimental results suggest that exploring the synergistic interaction of bioactive chemicals or nutrients in vivo could provide new insights in the development of therapeutic agents.

Despite its seriousness and interest to society, a therapeutic agent that could slow the aging process remains undeveloped. Many studies have reported lifespan extensions in animal experiments in ranges of approximately $10 \%$. Among the reported chemicals or nutrients extending the lifespans of the animals, rapamycin showed the greatest efficacy. However, rapamycin only extended animal lifespans from 9.3 to $16 \%$ depending on the experiments [47-49]. Although rapamycin impressively increases the lifespan, the efficacy of rapamycin on lifespan extension is still within an insignificant range of approximately $10 \%$, which poses the question as to whether the inhibition of the mTOR pathway by rapamycin $[50,51]$ is a key pathway in anti-aging or simply an anti-aging-related pathway. In this context, this work would shed light on understanding the aging process and developing an anti-aging therapeutic agent.

\section{Conclusions}

The resveratrol rice DJ526 is a genetically modified crop accumulated transgenic resveratrol in its grains, which provides upgrade potential against aging in wild-type ORR and Harwich D. melanogaster. Therefore, the resveratrol rice DJ526 leads to the generation of unexpectedly beneficial health effects, which can be used to extend the lifespan of $D$. melanogaster and prevent and/or treat various age-related diseases, bolstering hope that it may one day become possible to extend human lifespan.

Supplementary Materials: The following are available online at http://www.mdpi.com/2072-6643/11/8/1804/s1, Figure S1: The resveratrol rice DJ526 diet reduced vacuolar lesions in the brains of D. melanogaster. Table S1: Media compositions used to maintain the D. melanogaster ORR and Harwich strains.

Author Contributions: S.-T.H. conceived the project and designed the experiments; M.S.I., Y.Y.J., S.-H.B. and H.-J.C. performed the experiments and; M.S.I., H.-J.K. and S.-T.H. analyzed the data and wrote the manuscript; and S.-T.H. approved the final manuscript.

Funding: This work was supported by grants from the Next-Generation BioGreen 21 program (Project No. PJ014513012019), RDA and the Cooperative Research Program for Agriculture Science \& Technology Development (project no. PJ012806) Rural Development Administration, Republic of Korea.

Acknowledgments: We are grateful to Isaac A. Adedara at the Federal University of Santa Maria (Brazil) for providing wild-type ORR D. melanogaster. We are also grateful to Eukyung Hong at Boston College (US) for English proofreading.

Conflicts of Interest: The authors declare no conflict of interest. 


\section{References}

1. Dillin, A.; Gottschling, D.E.; Nyström, T. The good and the bad of being connected: The integrons of aging. Curr. Opin. Cell Biol. 2014, 26, 107-112. [CrossRef] [PubMed]

2. Tosato, M.; Zamboni, V.; Ferrini, A.; Cesari, M. The aging process and potential interventions to extend life expectancy. Clin. Interv. Aging 2007, 2, 401-412. [PubMed]

3. Bhullar, K.S.; Hubbard, B.P. Lifespan and healthspan extension by resveratrol. Biochim. Biophys. Acta 2015, 1852, 1209-1218. [CrossRef] [PubMed]

4. Burns, J.; Yokota, T.; Ashihara, H.; Lean, M.E.J.; Crozier, A. Plant foods and herbal sources of resveratrol. J. Agric. Food Chem. 2002, 50, 3337-3340. [CrossRef] [PubMed]

5. Howitz, K.T.; Bitterman, K.J.; Cohen, H.Y.; Lamming, D.W.; Lavu, S.; Wood, J.G.; Zipkin, R.E.; Chung, P.; Kisielewski, A.; Zhang, L.L.; et al. Small molecule activators of sirtuins extend Saccharomyces cerevisiae lifespan. Nature 2003, 425, 191-196. [CrossRef] [PubMed]

6. Guarente, L.; Picard, F. Calorie restriction-the SIR2 connection. Cell 2005, 120, 473-482. [CrossRef] [PubMed]

7. Bonkowski, M.S.; Sinclair, D.A. Slowing ageing by design: The rise of NAD+ and sirtuin-activating compounds. Nat. Rev. Mol. Cell Biol. 2016, 17, 679-690. [CrossRef]

8. Jarolim, S.; Millen, J.; Heeren, G.; Laun, P.; Goldfarb, D.S.; Breitenbach, M. A novel assay for replicative lifespan in Saccharomyces cerevisiae. FEMS Yeast Res. 2004, 5, 169-177. [CrossRef]

9. Miller, R.A.; Harrison, D.E.; Astle, C.M.; Baur, J.A.; Boyd, A.R.; de Cabo, R.; Fernandez, E.; Flurkey, K.; Javors, M.A.; Nelson, J.F.; et al. Rapamycin, but not resveratrol or simvastatin, extends life span of genetically heterogeneous mice. J. Gerontol. A Biol. Sci. Med. Sci. 2011, 66, 191-201. [CrossRef]

10. Sun, L.; Sadighi Akha, A.A.; Miller, R.A.; Harper, J.M. Life-span extension in mice by preweaning food restriction and by methionine restriction in middle age. J. Gerontol. A. Biol. Sci. Med. Sci. 2009, 64, 711-722. [CrossRef]

11. Baur, J.A.; Pearson, K.J.; Price, N.L.; Jamieson, H.A.; Lerin, C.; Kalra, A.; Prabhu, V.V.; Allard, J.S.; Lopez-Lluch, G.; Lewis, K.; et al. Resveratrol improves health and survival of mice on a high-calorie diet. Nature 2006, 444, 337-342. [CrossRef] [PubMed]

12. Viswanathan, M.; Kim, S.K.; Berdichevsky, A.; Guarente, L. A role for SIR-2.1 regulation of ER stress response genes in determining C elegans life span. Dev. Cell 2005, 9, 605-615. [CrossRef] [PubMed]

13. Chen, W.; Rezaizadehnajafi, L.; Wink, M. Influence of resveratrol on oxidative stress resistance and lifespan in Caenorhabditis elegans. J. Pharm. Pharmacol. 2013, 65, 682-688. [CrossRef] [PubMed]

14. Wood, J.G.; Rogina, B.; Lavu, S.; Howitz, K.; Helfand, S.L.; Tatar, M.; Sinclair, D. Sirtuin activators mimic caloric restriction and delay aging in metazoans. Nature 2004, 430, 686-689. [CrossRef] [PubMed]

15. Burnett, C.; Valentini, S.; Cabreiro, F.; Goss, M.; Somogyvári, M.; Piper, M.D.; Hoddinott, M.; Sutphin, G.L.; Leko, V.; McElwee, J.J.; et al. Absence of effects of Sir2 overexpression on lifespan in C. elegans and Drosophila. Nature 2011, 477, 482-485. [CrossRef] [PubMed]

16. Staats, S.; Wagner, A.E.; Kowalewski, B.; Rieck, F.T.; Soukup, S.T.; Kulling, S.E.; Rimbach, G. Dietary resveratrol does not affect life span, body composition, stress response, and longevity-related gene expression in Drosophila melanogaster. Int. J. Mol. Sci. 2018, 19, 223. [CrossRef] [PubMed]

17. Muhammad, M.S.; Magaji, R.A.; Mohammed, A.; Isa, A.S.; Magaji, M.G. Effect of resveratrol as caloric restriction mimetic and environmental enrichment on neurobehavioural responses in young healthy mice. Adv. Neuro. 2014, 2014, 1-7. [CrossRef]

18. Lagouge, M.; Argmann, C.; Gerhart-Hines, Z.; Meziane, H.; Lerin, C.; Daussin, F.; Messadeq, N.; Milne, J.; Lambert, P.; Elliott, P.; et al. Resveratrol improves mitochondrial function and protects against metabolic disease by activating SIRT1 and PGC-1alpha. Cell 2006, 127, 1109-1122. [CrossRef]

19. Pearson, K.J.; Baur, J.A.; Lewis, K.N.; Peshkin, L.; Price, N.L.; Labinskyy, N.; Swindell, W.R.; Kamara, D.; Minor, R.K.; Perez, E.; et al. Resveratrol delays age-related deterioration and mimics transcriptional aspects of dietary restriction without extending life span. Cell Metab. 2008, 8, 157-168. [CrossRef]

20. Barger, J.L.; Kayo, T.; Pugh, T.D.; Prolla, T.A.; Weindruch, R. Short-term consumption of a resveratrol-containing nutraceutical mixture mimics gene expression of long-term caloric restriction in mouse heart. Exp. Gerontol. 2008, 43, 859-866. [CrossRef] 
21. Baek, S.H.; Shin, W.C.; Ryu, H.S.; Lee, D.W.; Moon, E.; Seo, C.S.; Hwang, E.; Lee, H.S.; Ahn, M.H.; Jeon, Y.; et al. Creation of resveratrol-enriched rice for the treatment of metabolic syndrome and related diseases. PLoS ONE 2013, 8, e57930. [CrossRef] [PubMed]

22. Chung, H.J.; Sharma, S.P.; Kim, H.J.; Baek, S.H.; Hong, S.T. The resveratrol-enriched rice DJ526 boosts motor coordination and physical strength. Sci. Rep. 2016, 6, 23958. [CrossRef] [PubMed]

23. Baek, S.H.; Chung, H.J.; Lee, H.K.; D'Souza, R.; Jeon, Y.; Kim, H.J.; Kweon, S.J.; Hong, S.T. Treatment of obesity with the resveratrol-enriched rice dj-526. Sci. Rep. 2014, 4, 3879. [CrossRef] [PubMed]

24. Chung, H.J.; Lee, H.K.; Kim, H.J.; Baek, S.H.; Hong, S.T. Gene expression profiles and physiological data from mice fed resveratrol-enriched rice DJ526. Sci. Data 2016, 3, 160114. [CrossRef] [PubMed]

25. Khan, M.; Park, S.; Kim, H.J.; Lee, K.J.; Kim, D.H.; Baek, S.H.; Hong, S.T. The resveratrol rice DJ526 callus significantly increases the lifespan of Drosophila. Nutrients 2019, 11, 983. [CrossRef] [PubMed]

26. White, K.E.; Humphrey, D.M.; Hirth, F. The dopaminergic system in the aging brain of Drosophila. Front. Neurosci. 2010, 4, 205. [CrossRef] [PubMed]

27. Coulom, H.; Birman, S. Chronic exposure to rotenone models sporadic Parkinson's disease in Drosophila melanogaster. J. Neurosci. 2004, 24, 10993-10998. [CrossRef]

28. Cagan, R. Principles of Drosophila eye differentiation. Curr. Top. Dev. Biol. 2009, 89, 115-135.

29. Vauzour, D.; Camprubi-Robles, M.; Miquel-Kergoat, S.; Andres-Lacueva, C.; Bánáti, D.; Barberger-Gateau, P.; Bowman, G.L.; Caberlotto, L.; Clarke, R.; Hogervorst, E.; et al. Nutrition for the aging brain: Towards evidence for an optimal diet. Aging Res. Rev. 2017, 35, 222-240. [CrossRef]

30. Ungvari, Z.; Parrado-Fernandez, C.; Csiszar, A.; de Cabo, R. Mechanisms underlying caloric restriction and life span regulation: Implications for vascular aging. Circ. Res. 2008, 102, 519-528. [CrossRef]

31. Colman, R.J.; Beasley, T.M.; Kemnitz, J.W.; Johnson, S.C.; Weindruch, R.; Anderson, R.M. Caloric restriction reduces age-related and all-cause mortality in rhesus monkeys. Nat. Commun. 2014, 5, 3557. [CrossRef]

32. Liang, Y.; Liu, C.; Lu, M.; Dong, Q.; Wang, Z.; Wang, Z.; Xiong, W.; Zhang, N.; Zhou, J.; Liu, Q.; et al. Calorie restriction is the most reasonable anti-aging intervention: A meta-analysis of survival curves. Sci. Rep. 2018, 8, 5779. [CrossRef]

33. Kaeberlein, M.; Hu, D.; Kerr, E.O.; Tsuchiya, M.; Westman, E.A.; Dang, N.; Fields, S.; Kennedy, B.K. Increased life span due to calorie restriction in respiratory-deficient yeast. PLoS Genet. 2005, 1, e69. [CrossRef] [PubMed]

34. Barger, J.L. An adipocentric perspective of resveratrol as a calorie restriction mimetic. Ann. N. Y. Acad. Sci. 2013, 1290, 122-129. [CrossRef] [PubMed]

35. Barger, J.L.; Vann, J.M.; Cray, N.L.; Pugh, T.D.; Mastaloudis, A.; Hester, S.N.; Wood, S.M.; Newton, M.A.; Weindruch, R.; Prolla, T.A. Identification of tissue-specific transcriptional markers of caloric restriction in the mouse and their use to evaluate caloric restriction mimetics. Aging Cell 2017, 16, 750-760. [CrossRef] [PubMed]

36. Lopez, T.; Schriner, S.E.; Okoro, M.; Lu, D.; Chiang, B.T.; Huey, J.; Jafari, M. Green tea polyphenols extend the lifespan of male Drosophila melanogaster while impairing reproductive fitness. J. Med. Food 2014, 17, 1314-1321. [CrossRef] [PubMed]

37. He, Y.; Jasper, H. Studying aging in Drosophila. Methods 2014, 68, 129-133. [CrossRef]

38. Lee, S.H.; Lee, S.K.; Paik, D.; Min, K.J. Overexpression of fatty-acid- $\beta$-oxidation-related genes extends the lifespan of Drosophila melanogaster. Oxid. Med. Cell Longev. 2012, 2012, 854502. [CrossRef]

39. Mason, J.S.; Wileman, T.; Chapman, T. Lifespan extension without fertility reduction following dietary addition of the autophagy activator Torin1 in Drosophila melanogaster. PLoS ONE 2018, 13, e0190105. [CrossRef]

40. Zandveld, J.; van den Heuvel, J.; Zwaan, B.J.; Piper, M.D.W. Both overlapping and independent mechanisms determine how diet and insulin-ligand knockouts extend lifespan of Drosophila melanogaster. NPJ Aging. Mech. Dis. 2017, 3, 4. [CrossRef]

41. Bauer, J.H.; Goupil, S.; Garber, G.B.; Helfand, S.L. An accelerated assay for the identification of lifespan-extending interventions in Drosophila melanogaster. Proc. Nat. Acad. Sci. USA 2004, 101, 12980-12985. [CrossRef] [PubMed]

42. Colpo, A.C.; Lima, M.E.; da Rosa, H.S.; Leal, A.P.; Colares, C.C.; Zago, A.C.; Salgueiro, A.C.F.; Bertelli, P.R.; Minetto, L.; Moura, S.; et al. Ilex paraguariensis extracts extend the lifespan of Drosophila melanogaster fed a high-fat diet. Braz. J. Med. Biol. Res. 2018, 51, e6784. [CrossRef] [PubMed] 
43. Avanesian, A.; Khodayari, B.; Felgner, J.S.; Jafari, M. Lamotrigine extends lifespan but compromises health span in Drosophila melanogaster. Biogerontology 2010, 11, 45-52. [CrossRef] [PubMed]

44. Kõks, S.; Dogan, S.; Tuna, B.G.; González-Navarro, H.; Potter, P.; Vandenbroucke, R.E. Mouse models of aging and their relevance to disease. Mech. Aging Dev. 2016, 160, 41-53. [CrossRef] [PubMed]

45. Strait, J.B.; Lakatta, E.G. Aging-associated cardiovascular changes and their relationship to heart failure. Heart Fail. Clin. 2012, 8, 143-164. [CrossRef] [PubMed]

46. Bass, T.M.; Weinkove, D.; Houthoofd, K.; Gems, D.; Partridge, L. Effects of resveratrol on lifespan in Drosophila melanogaster and Caenorhabditis elegans. Mech. Aging Dev. 2007, 128, 546-552. [CrossRef] [PubMed]

47. Anisimov, V.N.; Zabezhinski, M.A.; Popovich, I.G.; Piskunova, T.S.; Semenchenko, A.V.; Tyndyk, M.L.; Yurova, M.N.; Rosenfeld, S.V.; Blagosklonny, M.V. Rapamycin increases lifespan and inhibits spontaneous tumorigenesis in inbred female mice. Cell Cycle 2011, 10, 4230-4236. [CrossRef] [PubMed]

48. Popovich, I.G.; Anisimov, V.N.; Zabezhinski, M.A.; Semenchenko, A.V.; Tyndyk, M.L.; Yurova, M.N.; Blagosklonny, M.V. Lifespan extension and cancer prevention in HER-2/neu transgenic mice treated with low intermittent doses of rapamycin. Cancer Biol. Ther. 2014, 15, 586-592. [CrossRef]

49. Fok, W.C.; Chen, Y.; Bokov, A.; Zhang, Y.; Salmon, A.B.; Diaz, V.; Javors, M.; Wood, W.H.; Zhang, Y.; Becker, K.G.; et al. Richardson A10.et al. Mice fed rapamycin have an increase in lifespan associated with major changes in the liver transcriptome. PLoS ONE 2014, 9, e83988. [CrossRef]

50. Johnson, S.C.; Rabinovitch, P.S.; Kaeberlein, M. mTOR is a key modulator of aging and age-related disease. Nature 2013, 493, 338-345. [CrossRef]

51. Fabrizio, P.; Pozza, F.; Pletcher, S.D.; Gendron, C.M.; Longo, V.D. Regulation of longevity and stress resistance by Sch9 in yeast. Science 2001, 292, 288-290. [CrossRef] [PubMed]

(C) 2019 by the authors. Licensee MDPI, Basel, Switzerland. This article is an open access article distributed under the terms and conditions of the Creative Commons Attribution (CC BY) license (http://creativecommons.org/licenses/by/4.0/). 